\title{
Definition, classification and visual aspects of diabetes mellitus, diabetic retinopathy and diabetic macular edema: A review of literature*
}

\section{AY Sukha ${ }^{\dagger}$ and A Rubin ${ }^{\dagger \dagger}$}

Optometric Science Research Group, Department of Optometry, APK, University of Johannesburg, PO Box 524, Auckland Park, 2006 South Africa

${ }^{\dagger}<$ baboo@icon.co.za>

$\dagger<$ arubin@uj.ac.za>

\section{Introduction}

Diabetes Mellitus (DM) is an interesting and complicated systemic disease. The World Health Organization (WHO) estimated that the global prevalence of DM would rise from $2.8 \%$ (171 million) to $4.4 \%$ (366 million) by 2030, with the most significant increases predicted in developing countries ${ }^{1}$. The development of DM immediately increases the patient's propensity to develop a number of irreversible acute and chronic complications. For example, impaired glucose tolerance and diabetic ketoacidosis are acute complications while chronic complications include macrovascular and microvascular anomalies. The macrovascular complications include cerebrovascular disease, coronary heart disease, and peripheral vascular disease. The microvascular complications include diabetic retinopathy (DR), diabetic neuropathy, and diabetic nephropathy ${ }^{2,3}$.

With the increase of prevalence in DM, the ocular manifestation of DM namely diabetic retinopathy (DR) will probably be the most frequent manifestation of a systemic disease encountered in primary-care optometry. Optometrists form part of the multi-disciplinary team aiding diabetic care. They are also often the first to examine patients with undiagnosed DM, or asymptomatic DR and diabetic macular edema $(\mathrm{DME})^{4,5}$. This possibly is even more so in South Africa, where optometrists are probably the most accessible eye care professionals to the public. General hospitals, clinics, and non-government organizations providing eye care are limited (approximately 25 government clinics to cater for 32 million people) and have very few optometrists and ophthalmologists available ${ }^{6}$.

In this paper the international and WHO definitions, classification and visual aspects of DM, DR and DME are presented. The ocular associations of DM and the visual aspects of DR and DME are also discussed. This information can be used to more accurately identify DM, DR and DME in high risk individuals.

\section{Definition of Diabetes Mellitus (DM)}

The World Health Organization defines DM as a metabolic disorder of multiple etiologies characterized by chronic hyperglycemia (raised glucose concentration) with disturbances of carbohydrate, fat and protein metabolism resulting from defects in insulin secretion, insulin action, or both ${ }^{7}$.

\section{Classification of DM}

Diabetes mellitus is classified into:

1. Type 1, or insulin dependent diabetes mellitus (IDDM);

2. Type 2, or non-insulin dependent diabetes mellitus (NIDDM);

3. Gestational diabetes mellitus (GDM), and

4. Other specific types of diabetes mellitus ${ }^{7}$.

*This paper is based on work by the first author for the degree MPhil in the Department of Optometry of the University of Johannesburg with the supervision of Professor A Rubin.

Received 5 July 2007, revised paper accepted 1 September 2007 


\section{Type 1 DM: Insulin dependent diabetes mellitus (IDDM)}

Type $1 \mathrm{DM}$ is divided into autoimmune and idiopathic DM. Both these types of DM result in insulin dependence and loss of pancreatic beta-cells $(\beta \text {-cells })^{7,8}$. The loss of these cells occurs gradually and few clinical symptoms present initially but acute episodes of diabetic ketoacidosis and hyperglycemia often result in initial diagnosis, particularly in children and adolescents. These patients may also have other autoimmune disorders such as Graves' disease, Hashimoto's thyroiditis, and Addison's disease 7

Type 1 autoimmune DM, is caused by $\beta$-cell dysfunction and/ or destruction. This leads to the loss of insulin secretion and absolute insulin deficiency. The etiology of $\beta$-cell dysfunction and destruction is still not well established. However, markers of $\beta$-cell dysfunction include islet cell auto-antibodies; autoantibodies to insulin; and auto-antibodies to glutei acid decarboxylase ${ }^{8-10}$. When these cells are damaged slowly and incompletely, it is known as latent autoimmune DM (LADA). This is a slowly progressive form of Type $1 \mathrm{DM}$ in adults, which masquerades as Type $2 \mathrm{DM}^{8,11}$.

Idiopathic Type $1 \mathrm{DM}$ occurs in patients who have insulinopenia and are prone to diabetic ketoacidosis, but have no evidence of autoimmunity. These patients periodically develop diabetic ketoacidosis ${ }^{7}$.

It has also been reported that both genetic susceptibility and environmental factors may account for the pathogenesis of all Type $1 \mathrm{DM}$. Various environmental triggers include viruses such as mumps and chicken pox, but thus far only congenital rubella syndrome has been conclusively associated with the disease ${ }^{7-9}$.

Insulin remains the main treatment in Type $1 \mathrm{DM}$, with islet transplantation and new immunosuppressive regimens as alternatives ${ }^{7,9}$.

\section{Type 2 DM: Non-insulin dependent diabetes mellitus (NIDDM)}

Type $2 \mathrm{DM}$ is a heterogeneous disorder, characterized by genetic and non-genetic factors as well as by an interaction between insulin resistance and pancreatic $\beta$-cell dysfunction ${ }^{7,12}$. The non-genetic factors of Type 2 DM include increasing age, high calorie intake, obesity, central adiposity, sedentary lifestyle, pregnancy, and low birth weight ${ }^{8,12,13}$.

The diagnosis of Type 2 DM usually occurs after the age of 40 years, although recently it has been found in younger children due to factors such as lack of exercise and obesity. Patients with Type 2 DM are frequently undiagnosed for many years because hyperglycemia is not severe enough to provoke very noticeable symptoms. Such patients are at a higher risk of developing macrovascular and microvascular complications ${ }^{7,12}$.

The following additional complications are experienced in Type 2 DM patients:

1. Acute hyperglycemia that causes patients to experience diabetic shock.

2. Diabetic ketoacidosis, which is more common in Type $1 \mathrm{DM}$, only arises in association with stresses of another illness such as an infection in Type 2 DM; and

3. Hyperglycemic hyperosmolar syndrome, which commonly develops in elderly Type 2 DM with progressive dehydration and hyperglycemia developing within days to weeks ${ }^{7,8,11}$.

Life style modifications, regular monitoring and compliance to treatment regimens are aids that improve glycemic control, and in turn reduce the development and progression of complications ${ }^{12,13}$.

\section{Gestational diabetes mellitus (GDM)}

GDM is a state of carbohydrate intolerance that develops during pregnancy. Women who develop Type $1 \mathrm{DM}$ during pregnancy and women with undiagnosed asymptomatic Type $2 \mathrm{DM}$ that is discovered during pregnancy are classified with GDM. GDM excludes women who have DM before they become pregnant ${ }^{7}, 14$. GDM may be viewed as:

1. An unidentified pre-existing disease, or

2. The unmasking of a compensated metabolic abnormality caused by the added stress of pregnancy, or

3. The direct consequence of the altered maternal metabolism stemming from the changing hormonal milieu ${ }^{8}$.

Formal systemic testing for GDM is usually done between 24 and 28 weeks of gestation (the end of the second trimester, and more commonly in the third trimester of pregnancy). GDM is asymptomatic, except 
when it is severe. High risk individuals include older women, those with a previous history of glucose intolerance, positive family history, obesity and any pregnant women who have elevated fasting, or casual blood glucose levels. Hyperglycemia resolves in most women after delivery but it also places them at increased risk of developing type 2 DM later in life. Early clinical recognition of GDM is important because there is an increased risk of delivery complications, and offspring are also at risk of macrosomia (birth weight in excess of $4 \mathrm{~kg}$ ), congenital abnormalities, and perinatal mortality ${ }^{7,8,14,15}$.

\section{Other specific types of DM}

These groups of DM include various etiologies in which the cause is established or at least partially known. The causes include known genetic defects of $\beta$-cell function or insulin action; diseases of the exocrine pancreas, drug, or chemical induced pancreatic changes; infections; genetic syndromes and other endocrinopathies. They also include diseases and conditions in which the incidence of diabetes is elevated, but a precise etiology is unknown ${ }^{7,8}$.

\section{Ocular associations of DM}

Common ocular associations of DM include cranial nerve palsies, poor corneal healing, decreased corneal sensitivity, open angle and neovascular glaucoma, iridoplegia, poor pupillary dilation, cataracts, refractive error shifts, DR, central retinal vein occlusion, and optic nerve papillopathy ${ }^{11,16}$.

\section{Cranial nerve palsies}

Cranial nerve palsies of the sixth, and more commonly third nerve, are usually associated with DM. Fourth nerve palsy is rare and may be seen with the third nerve palsy. These palsies are more prevalent in Type 2 DM patients over the age of 50 years. The onset occurs suddenly, with progression of the deficit lasting 1-2 days and full recovery over three to five months. In half the cases, the onset is associated with retro-orbital, or frontal pain, with the key feature of pupillary sparing. No specific treatment is required, other than prismatic or other compensation for diplopia as the ptosis recovers ${ }^{17,}{ }^{18}$. However, in a study conducted by Trigler et al, the presence of DR in Type 2 DM patients $(N=2229)$ with central nerve three, four, and six palsies, were significantly less compared to their controls. This result indicates a poor association between DR in Type 2 DM and diabetic ophthalmoplegia ${ }^{19}$.

\section{Decreased corneal sensitivity and healing}

The cornea is the most densely innervated part of the human body. In DM, increased glucose concentration in the cornea causes degeneration of small nerve fibres. This results in decreased corneal sensitivity, and changes in corneal nerve tortuosity ${ }^{20}$. The pathogenesis underlying these conditions includes suppressed cell division, altered barrier function, and deterioration of basal cell adhesion ${ }^{20-22}$. Generally, the corneal epithelium is maintained in diabetics, and corneal manifestations such as diabetic keratopathy, dry eye, or advanced keratoconjunctivitis sicca, only follow after corneal insults, such as intra-ocular surgery or mechanical injury ${ }^{21}$.

A further study reported that diabetic neuropathy of the cornea might also be an indicator of DR and other microvascular complications ${ }^{22}$. Morishige et al studied the corneal abnormalities in the basement membrane of 65 Type 2 DM subjects with different stages of DR, and compared them to 18 control subjects. Examination of the corneal layer did not show any marked differences in morphology, but the light scattering index of the basement membrane increased significantly greater than for the control subjects, and increased with the stages of DR. However, additional studies are required to determine if this non-invasive technique is sensitive enough to accurately assess the severity of diabetic complications ${ }^{22}$.

\section{Neovascular and Primary Open Angle Glaucoma (POAG)}

Glaucoma has been recognized by the WHO as one of the leading causes of blindness worldwide. It is the second leading cause of blindness in South Africa ${ }^{6}$. Compounding the problem are reports suggesting that neovascular glaucoma and POAG are more prevalent in $\mathrm{DM}^{16,23-26}$. However, other studies have shown no association between the two conditions ${ }^{27-29}$. Neovascular glaucoma is a complication of rubeosis iridis or iris neovascularization, where the patient usually presents with a chronically red painful eye ${ }^{16}$. It appears 
mainly in proliferative DR (PDR) and after vitrectomy. Usually these patients present with acuities less than 20/200 and IOP often exceeds $60 \mathrm{mmHg}^{30}$.

POAG is characterized by an IOP over $21 \mathrm{mmHg}$, an open anterior chamber angle, glaucomatous cupping, nerve fibre layer defects, and visual field loss ${ }^{16}$. Part of the controversy regarding the prevalence of POAG in DM exists because of variation of definitions in the above parameters. Zeiter and Shin ${ }^{24}$ reviewed charts of 144 randomly selected patients from a large glaucoma practice and found a statistically significant prevalence of DM $(p=0.0096)$ in patients with mainly inferior visual field defects in one or both eyes with POAG. Supporting evidence reported in the 20-year Nurses Health Study, where 76318 women, at least 40 years of age and without POAG, were recruited. Researchers positively associated Type 2 DM with POAG, with almost twice the relative risk ${ }^{23}$. Additionally, POAG is said to be six times more prevalent in Blacks (in certain age groups), and POAG appears and progresses approximately 10 years earlier in Blacks than in Whites $^{26}$. However, population based studies like the Baltimore Eye Study ${ }^{24}$, The Rotterdam Study ${ }^{27}$, The Visual Impairment Project ${ }^{29}$, and the Diabetes Audit and Research in Tayside study ${ }^{28}$ all found no association between POAG and DM.

\section{Pupillary abnormalities}

Clinical evaluation of the pupil provides information about the integrity and function of the iris, optic nerve, the posterior visual pathways and the third cranial and sympathetic nerves to the eye. For example, size (abnormality considered if pupils differ more than $0.5 \mathrm{~mm}$ in size, in light or dark conditions, constricted or dilated) and response abnormalities can be indicative of neurological defects (such as ptosis), lesions in afferent (retina or optic nerves) and efferent pathways (third and sympathic nerves ${ }^{31}$ ).

Poor pupillary dilation, tonic pupillary response (partial iridoplegia) and anisocoria result from autonomic neuropathy involving sympathetic nerve tissues in the iris with $\mathrm{DM}^{11,32}$. Some authors as early as 1967 reported recognizable changes in pupil reactions with $\mathrm{DM}^{32}$. For example, pupilographic analysis by Friedman et $a l^{32}$ indicated that 8 of $22 \mathrm{DM}$ subjects revealed abnormalities such as spastic miosis, and sluggish reaction to light. They also found that the mean latency period of pupillary contraction was significantly longer in subjects with advanced peripheral neuropathy. More recently ${ }^{33}, 76 \%$ of 332 Type 1 DM adolescents with mean DM duration 8.9 years were reported to have pupillary abnormalities (small initial pupil diameter, reduced reflex amplitude, and slow maximum constriction velocity). These limited reports conclude that pupillary abnormality is an important indicator of diabetic neuropathy.

\section{Cataracts}

In a summation of various studies there was evidence of an established relationship between DM and cataracts $^{34}$. Other studies reported a higher prevalence, and incidence (up to 50\%) of cataracts in DM patients compared to non-diabetics ${ }^{16,34,35}$. Approximately $4 \%$ of all cataracts are attributed to $\mathrm{DM}^{34}$.

$\mathrm{DM}$ is associated with mainly two types of cataracts, the senile cataract and the true diabetic cataract ${ }^{16}$. The diabetic cataract is further classified into cortical and posterior sub-capsular opacities ${ }^{11,34,35}$. In cortical cataracts, opacities spread along lens fibres from the equator to the center of the lens, whereas in the posterior type, opacities occur in front of the posterior lens capsule. This is due to the posterior migration of the epithelial cells of the lens ${ }^{16,35}$. The causes of these lens opacifications are primarily due to insolubilization of crystallines (for example proteins, $\mathrm{Ca}^{2}+$, fructose, sorbitol), and osmotic overhydration of the lens ${ }^{11,16,35}$.

The possible associations between various risk factors (blood pressure, body mass index, serum lipids, and smoking) and cataracts have been inconsistent. Excessive UV-B exposure and DM are casual risk factors of cortical cataracts; and steroidal treatment, DM, and ionizing radiation for posterior sub-capsular cataracts $^{34}$.

Accurate information regarding incidence and risk factors for diabetic cataracts is important to prevent visual impairment. Although in South African the main cause of blindness is age related cataract, the percentage of these people with DM is not well known ${ }^{6}$. 


\section{Central retinal vein occlusion (CRVO)}

CRVO is categorized into nonischemic (venous stasis occlusion) and ischemic (hemorrhagic) CRVO. There are very limited studies reporting on the prevalence or association of DM to CRVO. Approximately $15 \%$ of patients with CRVO will have $\mathrm{DM}^{11}$. More precisely it has been reported that ischemic CRVO is significantly prevalent in subjects with DM $(p=0.11)$ and arterial hypertension $(p=0.025)$. This prevalence increases further in the combined CRVO and hemi-central retinal vein occlusion group (DM: $p=0.005$, arterial hypertension: $p=0.02$ ). However, the authors concluded that the pathogenesis of CRVO is a multifactorial process and that the presence of a systemic disorder may or may not be a risk factor for $\mathrm{CRVO}^{36}$.

\section{Diabetic papillopathy}

Optic disc swelling occurs rarely in DM, and is reported in about only $0.4 \%$ of the diabetic population ${ }^{37}$. Diabetic papillopathy is often bilateral, asymptomatic, and is more frequently found in long standing juvenile diabetics ${ }^{11,38}$. Patients usually present with initial moderate reduced visual acuity, enlarged blind spots and approximately $70 \%$ present with macular edema ${ }^{38}$. Disc swelling and recovery of visual acuity often recovers within 90 days without any specific treatment ${ }^{11,39}$. Although studies do not necessarily associate diabetic papillopathy with DR, after a review of case studies it was concluded that subjects with diabetic papillopathy should be monitored closely for the presence or development of proliferative diabetic retinopathy $(\mathrm{PDR})^{37}$.

\section{Definition of Diabetic Retinopathy (DR)}

DR is composed of a characteristic group of lesions found in the retina of individuals having had DM for several years. The functional ocular sequelae may involve a blind, painful eye or enucleation. The development and progression of DR is often asymptomatic, and symptoms only occur if new vessels bleed (which is painless, unless the blood migrates to the anterior chamber of the eye), causing vision to be obscured. The presence and severity of DR may reflect, in varying degrees, complications of diabetes in other organs ${ }^{40}$.

\section{Classification of DR: The International DR Disease Severity Scale}

The International Clinical DR and DME Severity Scales are used in this paper to classify the various stages of retinopathy. This classification was proposed during an International Congress of Ophthalmology in Sydney in April 2002, with the aim to provide a consistent international classification system. This would then improve communication between optometrists, ophthalmologists, physicians and other eye care providers regarding severity of retinopathy. The diagnostic classification of DR includes the following stages: no apparent DR; mild non-proliferative DR; moderate non-proliferative DR; severe non-proliferative DR; and proliferative DR. The stages and observable findings are detailed in Table 1 below ${ }^{41}$.

Table 1. The classification and observable findings of DR according to the Diabetic Retinopathy Severity Scale (Reproduced with permission from Willkinson et al, and The Global Diabetic Retinopathy Project Group, 2003).

\begin{tabular}{|l|l|l|}
\hline Disease severity level & Abbreviations & Observable findings \\
\hline 1. No Apparent DR & NADR & No abnormalities \\
\hline 2. Mild Non-Proliferative DR & Mild NPDR & Microaneurysms only \\
\hline 3. Moderate Non-Proliferative DR & Moderate NPDR & $\begin{array}{l}\text { More than just microaneurysms but less than severe } \\
\text { nonproliferative DR }\end{array}$ \\
\hline 4. Severe Non-Proliferative DR & Severe NPDR & $\begin{array}{l}\text { Any of the following: } \\
\text { - More than 20 intraretinal hemorrhages in each of 4 } \\
\text { quadrants } \\
\text { - definite venous beading in 2+ quadrants } \\
\text { - prominent intraretinal microvascular } \\
\text { abnormalities in 1+ quadrant } \\
\text { - No signs of proliferative DR }\end{array}$ \\
\hline 5. Proliferative DR & PDR & $\begin{array}{l}\text { One or more of the following: } \\
\text { - neovascularization } \\
\text { - vitreous/ preretinal hemorrhage }\end{array}$ \\
\hline
\end{tabular}




\section{Definition of DME}

DME is defined as the presence of any retinal thickening or hard exudates within one disc diameter $(1500 \mu \mathrm{m})$ of the center of the fovea, or one disc diameter from the center of the macula ${ }^{16}$.

\section{Classification of DME: The International DME Disease Severity Scale}

According to the DME Disease Severity Scale ${ }^{41}$, the condition is classified into absent, mild, moderate, and severe DME (Table 2). Mild DME occurs when there is some retinal thickening or hard exudates in the posterior pole distant from the macula. Moderate, where retinal thickening or hard exudates are approaching the center of the macula, but not involving the center, and severe where retinal thickening and hard exudates involve the center of the macula ${ }^{41}$.

Table 2. Classification of DME according to the DME Disease Severity Scale. (Reproduced with permission from Willkinson et al, and The Global Diabetic Retinopathy Project Group, 2003).

\begin{tabular}{|l|l|}
\hline Severity Level & Observable findings \\
\hline DME apparently absent & No apparent retinal thickening or hard exudates in posterior pole. \\
\hline DME apparently present & Some apparent retinal thickening or hard exudates in posterior pole. \\
\hline If DME is present, it can be categorized as follows: \\
\hline Mild DME & $\begin{array}{l}\text { Some apparent retinal thickening or hard exudates in posterior pole but distant } \\
\text { from the center of the macula. }\end{array}$ \\
\hline Moderate DME & $\begin{array}{l}\text { Retinal thickening or hard exudates approaching the center of the macula but not } \\
\text { involving the center. }\end{array}$ \\
\hline Severe DME & Retinal thickening or hard exudates involving the center of the macula. \\
\hline
\end{tabular}

\section{Aspects of vision loss in DR and DME}

According to the WHO International Classification of Visual Standards, four main aspects of visual loss can be identified (Figure 1). Two aspects refer to the organ system, and the other two to the individual ${ }^{42}$. The organ system is divided into structural and functional changes, where medical and surgical interventions minimize structural impairments of various disorders. In vision, the term visual function is used to refer to the impairment aspect. This is assessed quantitatively, for example by visual acuity (VA), contrast sensitivity (CS), colour vision (CV) et cetera. Here intervention of visual aids or devices can help improve the ability to perform various activities. The remaining two aspects refer to the individual, and are divided into skills and abilities, and social consequences of the person. The term functional vision is used in this aspect to refer to visual abilities as they are needed for tine proper performance of Activities of Daily Living (ADL), which is measured by questionnaires. Education, training, and work place adaptations help reduce the social and economic impact of the loss of ability. Intervention at various stages makes rehabilitation possible and can predict changes from one aspect to another to a certain extent.

\begin{tabular}{|c|c|c|c|c|}
\hline \multicolumn{3}{|c|}{ The Organ } & \multicolumn{2}{|c|}{ The Person } \\
\hline Aspects: & Structural & Functional Change & $A D L$ & Social \\
\hline Terms: & Health Condition & Organ Function & Skills/Abilities & Participation \\
\hline Limitations: & Disorder/Injury & Impairment & Ability loss & Handicap \\
\hline VISION: & Eye Health & Visual Function & Functional Vision & \\
\hline TEST & & $\mathrm{VA}, \mathrm{CS}, \mathrm{CV}$ & Questio & ski \\
\hline Interventions & s: Medical/Surgical & Visual Aids/Devices & Education/Trainin & \\
\hline
\end{tabular}

Figure 1. The four main aspects of vision loss classified into terms, limitations, application to vision, performance tests and interventions. ADL: Activities of Daily Living; VA: visual acuity; CS: contrast sensitivity; CV: colour vision. The table has been adapted from the WHO, International Classification of Visual Standards (2002). 


\section{Visual Acuity (VA)}

Direct comparison of studies that have reported the prevalence and effects of DR and DME with regard to ranges of acuity loss are difficult, due to lack of uniformity in acuity definition and other methodology. However, the following few studies do provide some valuable information regarding VA loss associated with DR and DME. In the USA, visual impairment is defined as having the best corrected VA of $\leq 6 / 12$ and blindness as VA $\leq 6 / 60$ in the better seeing- eye ${ }^{43}$. In the American National Health Interview Survey, DR was reported as one of the leading causes of visual impairment and blindness. The others being age-related eye diseases ${ }^{43}$. The only other country that reported such extensive loss of vision due to DR and DME was India where Rema, Ponnaiya and Mohan ${ }^{44}$ reported decreased visual acuity due to DR in $44.5 \%$ in a cohort of 1062 visually impaired Type 2 DM subjects, with DME accounting for $78.8 \%$ of the decrease in VA among the diabetes related causes.

Conversely, smaller independent clinical studies in the USA ${ }^{45}$, Sweden ${ }^{46}$, Nigeria ${ }^{47}$ and the United Kingdom Prospective Diabetes Study (UKPDS) ${ }^{48}$ showed that the prevalence of visual impairment (between $12 \%-40 \%$ ) and blindness (between 3\%-25\%) caused by DR and DME in diabetic patients to be variable, and results depended on the size of the samples, which were sometimes small.

The Wisconsin Epidemiological Study of Diabetic Retinopathy (WESDR) is probably the most reliable study reporting visual loss in the diabetic population because it was primary care based, it had an adequate sample size, a lengthy follow up, and they used standard protocols for measuring VA. The cumulative 14year incidences of visual impairment, doubling of the visual angle and blindness were reported at $12.7 \%$, $14.2 \%$ and $2.4 \%$ respectively. The doubling of visual angle was further associated independently with DR, glycated haemoglobin $(\mathrm{HbAlc})$, proteinuria and age ${ }^{49}$. Additionally, in the Early Treatment Diabetic Retinopathy Study (ETDRS) randomized controlled clinical trail, demographic, clinical, and laboratory characteristics of 3711 subjects were assessed. Of these 1444 subjects were diagnosed with Type 1 DM and 2267 with Type 2 DM. Results of this study revealed the most astounding association between VA (using standardized ETDRS letter charts at 4 and 1 metres where necessary) and mortality in both Type 1 and Type 2 DM subjects. They reported that amputation and poor VA remained statistically significantly associated with mortality in patients with Type $1 \mathrm{DM}^{50}$. Additionally, visual impairment and not the degree of DR were related to mortality. In Type 2 DM all complications including DR and VA were independently associated with mortality even after baseline risk factors were controlled.

In summary, we can conclude that VA does deteriorate significantly with increasing severity of DR and more so with DME. Additionally there is a possibility that VA in diabetics might be independently related to mortality. However, consistent estimates of vision loss dxe to DR and DME are as yet undetermined.

\section{Refractive Status}

Shifts or fluctuation in refractive status in DM are often acute and relate to the levels of hyperglycemia present. Accumulation of water in the ocular lens and the amount of sorbital by-products alters the refractive indices of the eye. Changes in the tear layer and cornea of diabetics may also be additional factors. The stability of refractive status is determined largely through the stability of the serum glucose and fluctuation of less than $80-100 \mathrm{mg} / \mathrm{dl}$ for the serum glucose levels over a three to four week period is recommended for stabilization of refractive error ${ }^{11}$. In the Beaver Dam Eye Study ${ }^{51}$ the 10 -year change in spherical equivalent refraction was determined in 2937 subjects. They concluded using a multivariate model that the presence of DM tended to cause a larger shift in hyperopia. The Visual Impairment Project (Australia) defined myopia as a best corrected minus spherical equivalent power greater than $-0.50 \mathrm{D}$. McKay, McCarty and Taylor reported that myopia was not significantly correlated with $\mathrm{DR}^{29}$. Similarly, Espiritu and Sy, found no significant association between DME and myopia $(p=0.742)^{52}$.

\section{Contrast sensitivity}

Contrast sensitivity has been shown to provide psychophysical information regarding visual function detecting subtle or insidious disease processes which may have not affected visual acuity as clinically measured. Measurements of contrast sensitivity can also be used to monitor the course of a disease and can be used to understand how patients with reduced contrast function when engaged in various visual 
activities $^{31,53}$. Although both hue discrimination and contrast sensitivity reflect macula function, their exact physiological relationship to DR and DME has not been fully explained ${ }^{53}$.

There is evidence regarding the association between abnormal contrast sensitivity and the presence of $\mathrm{DR}^{54,55}$. There seems to be evidence that a significant increase in contrast sensitivity thresholds is more marked in subjects with PDR and thresholds are also elevated significantly in the background DR group when compared to those without $\mathrm{DR}^{54}$. Another study found an association at 6 cycles per degree $(\mathrm{c} / \mathrm{d})$ between contrast sensitivity and the grade of $\mathrm{DR}^{56}$. However, Arend et al, reported abnormal contrast sensitivity to spatial frequencies of 6 and $12 \mathrm{c} / \mathrm{d}$ in DM subjects with VA 20/25 or better and without clinical significant macular edema ${ }^{57}$.

Unfortunately the variety of measurement procedures, the calibration required and the equipment used sometimes preclude direct or simple comparisons of the results from different studies. These studies indicate that contrast sensitivity might be a sensitive indicator of early visual impairment and should be used in conjunction with other tests to identify early DR and DME.

\section{Colour vision}

DM has been associated with the acquisition of a specific type of color defect involving the blue-yellow (tritan-like) system. Reports of colour vision suggest that the short wavelength-sensitive cone systems are more susceptible to damage in a variety of retinal disease where changes are more confined to alterations of the inner retina ${ }^{58,59}$.

It is well documented that patients with Type $1 \mathrm{DM}$ can show alterations in their colour perception even before the onset of $\mathrm{DR}^{59,60}$. In the ETDRS, approximately 50\% (approximately 1350 ) of patients at base line had abnormal hue discrimination. DME severity, age, and the presence of new vessels were factors most strongly associated with impaired colour discrimination ${ }^{59}$. These authors, using the FM-100 hue test, also reported increased correlation between severity of macula edema with total error scores, resulting in more prominent tritan defects. In another study after cluster analysis of the Fourier series derived from the FM 100 -hue test, 13 patterns of impaired hue discrimination were found ${ }^{61}$. A tritan defect was observed in $26 \%$ of subjects, $10 \%$ had a generalized decrease in hue discrimination, and the remainder had a combination of tritan- and deutan-like defects or protan- and tritan-like defects. These results represent, for example, male subjects with congenital red-green defect, along with tritan changes caused by DME ${ }^{61}$.

\section{Intra ocular pressure (IOP)}

Very few studies have assessed the relationship between DM, hyperglycemia, DR and IOP ${ }^{25,62,63}$. For instance, in a cross-sectional study, Oshitari ${ }^{62}$ divided DM patients into mild ( $\left.\mathrm{HbA} 1 \mathrm{c} \leq 6.5 \%\right)$, moderate $(6.5<\mathrm{HbA} 1 \mathrm{c}<8 \%)$ and severe (HbA1c $\geq 8 \%)$ hyperglycemia groups. None of these patients had DR, secondary glaucoma or a history of glaucoma. Results indicated that the mean IOP in the mild group was statistically lower than that of the severe group $(p=0.013)$. They concluded chronic hyperglycemia is associated with increased IOP. Other studies associated low IOP with DR and haemorrhagic glaucoma respectively ${ }^{25,63}$. Madsen showed that IOP fell in parallel with the development of DR ${ }^{63}$. He classified DR in the following stages: no proliferation, naked vessels, connective tissue and dense vascular proliferation (active stage), dense connective tissue with contracture and decrease in vascular system (regression stage) and fundus not seen. Significant differences were seen in stages of no proliferation and active stage

$(p<0.01)$, and regression stages $(p<0.001)$ and between naked vessel and active stage $(p<0.05)$, regression stages $(p<0.01)$. Additionally he found that in some cases, hypotonia was followed by neovascularization of the iris and anterior chamber, and subsequently by haemorrhagic glaucoma. Similarly, Soares et al, significantly associated optic disc haemorrhages in glaucoma with the presence of DM, and relatively lower IOP during follow-ups visits ${ }^{25}$.

These studies suggest that an increased IOP might be observed in DM patients with chronic hyperglycemia, but a lower IOP in DM patients with DR and haemorrhagic glaucoma is also likely. 


\section{Activities of daily living: quality of life}

Quality of life is defined as a multidimensional concept including physical (disease symptoms and their treatment); functional (mobility, quality of life, activity levels); social (interpersonal relationships), and psychological (emotional status, cognitive function, and happiness) dimensions. Of these, functional limitation is one of the most important measurements, as it represents the impact of visual impairment on individuals in their daily lives ${ }^{64}$. This has led to the development of numerous questionnaires which primarily aim to identify these restrictions in daily living participation caused by visual impairment (in this case visual impairment caused by DR or DME). By identifying these impairments, strategies and interventions can be implemented to improve, and monitor rehabilitation ${ }^{64,65}$.

Diabetic persons encounter a unique set of visual challenges, for example, using colour to identify glucose levels, visual fluctuations, and determining correct insulin dosage levels ${ }^{66-68}$. Therefore, optometrists can also aid in providing visual rehabilitation information to assist patients in optimizing their remaining vision. For example, if one of the main visual needs is being able to read a newspaper or to read insulin dosages, ruler type magnifiers or clip-on magnifiers can be prescribed. For monitoring blood glucose levels, meters with large print displays or audible read outs can be made available. Lastly because vision loss can contribute to the onset of depression, counseling can also be beneficial for these patients, especially in support groups that provide an encouraging atmosphere ${ }^{69}$. The aim therefore is to identify specific areas that vision rehabilitation services can target.

Only one questionnaire, the Retinopathy Treatment Satisfaction Questionnaire (RetTSQ), was aimed specifically at the diabetic person, but only for the evaluation of currently available and anticipated new therapies ${ }^{67}$. Only two studies recruited subjects with DR, the one using the National Eye Institute Visual Function Questionnaire and the other, the Impact of Vision Impairment Questionnaire (IVI) ${ }^{70,71}$. In a cross sectional population based study of 4774 participants, with the use of the National Eye Institute Visual Function Questionnaire subjects with DR reported low scores in near and distance tasks, which were not attributed to decreased visual acuity ${ }^{70}$. Contrast sensitivity, glare sensitivity, and colour vision were factors that might have contributed to these scores. These DR subjects also showed a significant decrease in driving scores, compared to subjects with no eye disease ${ }^{70}$. In the IVI questionnaire the greatest restriction of the 45 DR subjects with VA worse than 6/12, were found in the leisure and work, mobility, and consumer and social interaction domains ${ }^{71}$.

\section{Conclusion}

The aim of this paper was to present a literature review of current definitions, classifications and visual or ocular aspects of DM, DR and DME. It is suggested that the WHO classification of DM, and the international classification of DR and DME be used in further studies. This would firstly, allow for direct comparison of results between both local and international studies, and secondly, provide better communication between optometrists, ophthalmologists and other health care providers regarding DR severity.

The review on the various ocular associations of DIM, and visual aspects of DR and DME can be used to form visual profiles which can aid in identifying and managing DR and DME. For example, a 40-year old patient with reduced contrast sensitivity and VA presenting with POAG should be evaluated for the presence of DM and/or DR and DME. Additionally, optometrists and ophthalmologists can provide some visual rehabilitation education to improve quality of life for these patients. For example, by informing patients to clear obstacles and pathways, or to paint white lines on the edge of steps, or to add handrails which would all ease orientation and mobility.

This review also highlights the need for further research required in both ocular and visual aspects regarding DR and DME, especially in South Africa.

\section{References}

1. Wild S, Roglic G, Green A, Sicree R, King H. Global prevalence of diabetes. Diabetes Care $2004271047-1051$.

2. Bailes BK. Diabetes mellitus and its chronic complications. AORN Journal 2002 76 265-282.

3. Phillip P, Popplewell P. Diabetes: what's new in type 2? Modern Medicine of South Africa 1997 July 54-63. 
4. Verma L, Gunjan P, Terwari HK, Gupta SK, Murthy GVS, Sharma N. Screening for diabetic retinopathy by nonophthalmologist: an effective public health tool. Acta Ophthalmologica Scandinavica 2003 81 373-377.

5. Lightstone A. Review of the effectiveness of optometrists in the monitoring of diabetic retinopathy. Public Health Research Report 95, Essex, 1999.

6. Sacharowitz H. Visual impairment in South Africa: achievements and challenges. The South African Optometrist 200564 139-149.

7. World Health Organisation (WHO). Definition, diagnosis and classification of diabetes mellitus and its complications. WHO 1999 1-50.

8. LeRoith D, Taylor SI, Olefsky JM. Diabetes Mellitus. A Fundamental and Clinical Text. $2^{\text {nd }}$ edition. Philadelphia: Lippincott Williams and Wilkins, 2000.

9. Devendra D, Liu E, Eisenbarth GS. Type 1 diabetes: recent developments. British Medical Journal $2004328750-754$.

10. Kousta E, Lawrwnce NJ, Anyaoku V, Johnston DG, McCarthy MI. Prevalence and features of pancreatic islet cell autoimmunity in women with gestational diabetes from different ethnic groups. British Journal of Obstetrics and Gynaecology 2001108 716-720.

11. Blaustein BH. Ocular Manifestations of Systemic Diseases. New York: Churchill Livingston Inc, 1994.

12. Department of Health (DOH). National Programme for the Control and Management of Diabetes Type 2 at Primary Level. South African Communication Service, Department Of Health, April 1998.

13. Horton ES. NIDDM - the devastating disease. Diabetes Research and Clinical Practice 199528 S3-11.

14. Cianni DG, Volpe L, Lencioni C, Miccoli R, Cuccuru I, Ghio A, Chatzianagnostou K, Bottone P, Teti G, Del Prato S, Benzi L. Prevalence and risk factors for gestational diabetes assessed by universal screening. Diabetes Research and Clinical Practice 200362 131-137.

15. Zargar AH, Sheik MI, Bashir MI, Masoodi SR, Laway BA, Wani AI, Bhat MH, Dar FA. Prevalence of gestational diabetes mellitus in Kashmiri women from the Indian subcontinent. Diabetes Research and Clinical Practice 2004 66 139-145.

16. Kanski JJ. Clinical Ophthalmology. $3^{\text {rd }}$ edition. Oxford: Butterworth Heinemann, 1994.

17. Llewelyn JG. The diabetic neuropathies: types, diagnosis, and management. Journal of Neurology, Neurosurgeons and Psychiatry 200374 S15-19.

18. Said G. Diabetic neuropathy: an update. Journal of Neurology 1996243 431-440.

19. Trigler L, Siatkowski RM, Oster AS, Feuer WJ, Betts CL, Glaser JS, Schatz NJ, Farris BK, and Flynn HW. Retinopathy in patients with diabetic ophthalmoplegia. Ophthalmology 2003110 1545-1550.

20. Hossain P, Sachdev A, Malik RA. Early detection of diabetic peripheral neuropathy with corneal confocal microscopy. The Lancet 2005366 1340-1343.

21. Karpecki PM. Diabetes care: Don't forget the cornea. Review of Optometry 2004 September 136-137.

22. Morishige N, Chikama TI, Sassa Y, Nishiaa T. Abnormal light scattering detected by confocal biomircoscope at the corneal epithelial basement membrane of subjects with Type 2 diabetes. Diabetologia 200144 340-345.

23. Pasquate LR, Kang JH, Manson JE. Prospective study of type 2 diabetes mellitus and risk of primary open-angle glaucoma in women. Ophthalmology 2006113 1081-1086.

24. Zeiter JH, Shin DH. Diabetes in primary open angle glaucoma patients with inferior visual field defects. Graefe's Arch Clinical and Experimental Ophthalmology 1994232 205-210.

25. Soares AS, Artes PH, Andreou P, Leblanc RP, Chauhan BC, Nicolela MT. Factors associated with optic disc hemorrhages in glaucoma. Ophthalmology 200411 1653-1657.

26. Racette L, Wilson MR, Zangwill LM, Weinreb RN, Sample PA. Primary open-angle glaucoma in Blacks: A Review. Survey of Ophthalmology 200348 295-305.

27. de Voogd S, Ikram MK, Wolfs RCW, Jansonius NM, Witteman JCM, Hofman A, de Jong PTVM. Is Diabetes Mellitus a risk factor for open-angle glaucoma. Ophthalmology 2006113 1827-1831.

28. Ellis JD. Glaucoma incidence in an unselected cohort of diabetic patients: is diabetes mellitus a risk factor for glaucoma. American Journal of Ophthalmology 2001 February 286.

29. McKay R, McCarty CA, Taylor HR. Diabetic retinopathy in Victoria, Austrialia: the Visual Impairment Project. British Journal of Ophthalmology 200084 865-870.

30. Johnson GK, Minassian DC, Weale RA, West SK. The Epidemiology of Eye Disease. $2^{\text {nd }}$ edition. London: Arnold, 2003.

31. Eskridge JB, Amos JF, Bartlett JD. Clinical Procedures in Optometry. Philadelphia: JB Lippincott Company, 1991.

32. Friedman SA, Feinberg P, Podolak E, Bedell RH. Pupillary abnormalities in diabetic neuropathy. Annuals of Internal Medicine 196767 971-983.

33. Donaghue KC, Margan SH, Chan AK, Holloway B, Silink M, Rangel T, Bennetts B. The association of aldose icductase gene (AKR1B1) polymorphins with diabetic neuropathy in adolescents. Diabetic Medicine 2005 22 1315-1320.

34. Robman L, Taylor H. External factors in the development of cataract. Eye 200519 1074-1082.

35. Biswas S, Harris F, Singh J, Phoenix D. Role of carpins in diabetes mellitus-induced cataractogenesis: a mini review. Molecular and Cellular Biochemistry 2004 February 1-9.

36. Hayreh SS, Zimmerman B, McCarthy MJ, Podhajsky P. Systemic diseases associated with various types of retinal vein occlusion. American Journal of Ophthalmology 2001 131 61-77.

37. Stransky TJ. Diabetic papillopathy and proliferative retinopathy. Graefe's Archive for Clinical and Experimental Ophthalmology 1986224 46-50. 
38. Mansour AM, El-Dairi MA, Shehab M, Shahin HK, Shaaban JA, Antonios SR. Periocular corticosteriods in diabetic papillopathy. Eye 200519 45-51.

39. Fraser-Bell S, Capo M. Optic disc swelling in an adolescent with insulin dependent diabetes mellitus. Clinical and Experimental Ophthalmology 200230 434-436.

40. Alexander KL. The Lippincott Manual of Primary Eye Care. Philadelphia: J.B. Lippincott Company, 1995.

41. Willkinson CP, Ferris FL, Klein RE, Lee PP, Agardh CD, Davis M, Dills D, Kampik A, and the Global Diabetic Retinopathy Project Group. Proposed International Clinical Diabetic Retinopathy and Diabetic Macular Edema Disease Severity Scales. Ophthalmology 2003110 1677-1682.

42. International Council of Ophthalmology. Visual Standards: Aspects and ranges of vision loss with emphasis on population surveys. $29^{\text {th }}$ International Congress of Ophthalmology, 2002 April.

43. Saaddine J, Benjamin S, Pan L, Venkat Narayan KM, Tierney E, Kanjilal S, Geiss L. Prevalence of visual impairment and selected eye diseases among persons aged $>50$ years, with and without diabetes, United States, 2002. Morbidity and Mortality Report 200453 1069-1071.

44. Rema M, Ponnaiya M, Mohan V. Prevalence of retinopathy in non insulin dependent diabetes mellitus at a centre in Southern India. Diabetes Research and Clinical Practice 199634 29-36.

45. Roy MS, Klein R, O'Colmain BJ, Klein BE, Moss SE, Kempen JH. The prevalence of diabetic retinopathy among adult type 1 diabetic persons in the United States. Arch Ophthalmology 2004122 546-551.

46. Lovestam-Adrian M, Agardh CD, Torffvit O, Agardh E. Diabetic retinopathy, visual acuity, and medical risk indicators. A continuous 10-year follow up study in Type 1 diabetic patients under routine care. Journal of Diabetes and Its Complications 200115 287-294.

47. Nwosu SN. Low vision in Nigerians with diabetes mellitus. Documenta Ophthalmologica 2000 101 51-57.

48. Kohner EM, Aldington SJ, Stratton IM and the United Kingdom Prospective Diabetes Study Group. United Kingdom Prospective Diabetes Study, 30. Arch Ophthalmology 1998116 297-303.

49. Moss SE, Klein R, Klein BE. The 14-year incidence of visual loss in a diabetic population. Ophthalmology 1998 105 998 1003.

50. Cusick M, Meleth AD, Agron E, Fisher MR. Associations of mortality and diabetes complications in patients with type 1 and type 2 diabetes: Early Treatment Diabetic Retinopathy Study report no. 27. Diabetes Care 2005 28 617-626.

51. Lee KE, Klein BE, Klein R, Wong TY. Changes in refraction over a 10-years in an adult population: The Beaver Dam Eye Study. Investigative Ophthalmology and Visual Science 2002 \$3 2566-2571.

52. Espiritu RB, Sy GT. Fluorescein angiographyically evident in diabetic maculopathy. Clinical Hemorheology and Microcirculation 200329 357-365.

53. Gardner TW, Antonetti DA, Barber AJ, LaNoue KF, Levison SW and The Penn State Retina Research Group. Diabetic retinopathy: more than meets the eye. Survey Of Ophthalmology 200247 S253-262.

54. Ghafour IM, Foulds WS, Allan D, McClure E. Contrast sensitivity in diabetic subjects with and without retinopathy. British Journal of Ophthalmology 198266 492-495.

55. Sokol S, Maskowitz A, Skarf B, Evans E, Molitch M, Senior B. Contrast sensitivity in diabetics with and without diabetic retinopathy. Arch Ophthalmology 1985103 51-54.

56. Brinchmann HO, Bangstad HJ, Hultgren S, Fletcher R, Dahl JK, Hanssen KF, Sandvik L. Psychophysical visual function, retinopathy, and glyceamic control in insulin-dependent diabetics with normal visual acuity. Acta Ophthalmology $1993 \mathbf{7 1}$ 230-237.

57. Arend O, Remky A, Evans D, Stuber R, Harris A. Contrast sensitivity loss is coupled with capillary dropout in patients with diabetes. Investigative Ophthalmology and Visual Science 199738 1819-1824.

58. Afrashi F, Erakgun T, Kose S, Ardic K, Mentes J. Blue-on-yellow perimetry versus achromatic perimetry in type 1 diabetes without retinopathy. Diabetes Research and Clinical Practice 200361 7-11.

59. Fong DS, Barton FB, Bresnick GH. Impaired color vision associated with diabetic retinopathy: ETDRS Report 15. American Journal of Ophthalmology 1999128 612-617.

60. Verrotti A, Lobefalo L, Chiarelli F, Mastropasqua L, Ćiancaglini M, Morgese G. Colour vision and persistent microalbuminuria in children with type 1 diabetes mellitus: a longitudinal study. Diabetes Research and Clinical Practice 199530 125-130.

61. Barton FB, Fong DS, Knatterud GL and the ETDRS Research Group. Classification of Fransworth-Munsell 100-hue test results in the early treatment diabetic retinopathy study. American Journal of Ophthalmology 2004 138 119-124.

62. Oshitari T. Effect of chronic hyperglycemia on intraocular pressure in patients with diabetes. American Journal of Ophthalmology 2006 In Press (uncorrected proof) 1-2.

63. Madsen PH. Ocular findings in 123 patients with proliferative diabetic retinopathy. Documenta Ophthalmologica 197129 345-349.

64. Stelmack J. Quality of life of low-vision patients and outcomes of low vision rehabilitation. Optometry and Vision Science 200178 335-342.

65. Weih LM, Hassel JB, Keeffe J. Assessment of the Impact of Vision Impairment. Investigative Ophthalmology and Visual Science 200243 927-935.

66. Bourne JH. Experiences of people in Wales who are registered blind or partially sighted due to diabetic retinopathy. International Congress Series 20051282 31-34. 
67. Woodcock A, Ploywright R, Kennedy-Martin T Hirsch A, Ffyche T, Bradley C. Development of the new Retinopathy Treatment Satisfaction Questionnaire (RetTSQ). International Congress Series 2005 1282 342-346.

68. Rosenthal JL. Special problems of people with diabetes and visual impairment. Journal of Visual Impairment and Blindness 1993 November 331-333.

69. Cooke JB, Cochrane AL. A practical guide to low vision management of patients with diabetes. Clinical and Experimental Optometry 200184 155-161.

70. Broman AT, Munoz B, Rodriguez J, Sanchez R, Quigley, Klein R, Snyder R, West SK. The impact of visual impairment and eye disease on vision related quality of life in a Mexican-American population: Proyecto VER. Investigative Ophthalmology and Visual Science 200443 1-11.

71. Lamourex E, Hassell JB, Keeffe JE. The impact of diabetic retinopathy on participation in daily living. Arch Ophthalmology 2004122 84-88. 